\title{
Multilayer Brightness Temperature Tracing Method for Rough Surface Scene Simulation in Passive Millimeter-Wave Imaging
}

\author{
Chuan Yin, ${ }^{1,2}$ Ming Zhang, ${ }^{1,2}$ and Yaming $\mathrm{Bo}^{1,2}$ \\ ${ }^{1}$ School of Electronic Science and Engineering, Nanjing University of Posts and Telecommunications, Nanjing 210003, China \\ ${ }^{2}$ State Key Laboratory of Millimeter Waves, Southeast University, Nanjing 210096, China \\ Correspondence should be addressed to Yaming Bo; ymbo@njupt.edu.cn
}

Received 29 February 2016; Accepted 15 September 2016

Academic Editor: Atsushi Mase

Copyright (C) 2016 Chuan Yin et al. This is an open access article distributed under the Creative Commons Attribution License, which permits unrestricted use, distribution, and reproduction in any medium, provided the original work is properly cited.

\begin{abstract}
Simulation in passive millimeter-wave (MMW) imaging of rough surfaces is an indispensable step in the simulation in passive radiation imaging, especially for the rough surfaces of different roughness surfaces. However, little attention has been paid to the simulation of rough surface; based on the existing model of brightness temperature tracing described in previous work, diffused reflection of the rough surface is taken into account in the improved model which is presented in this paper. In the paper, the brightness temperature tracing model of different roughness surfaces has been established. Then, we present a method called multilayer brightness temperature tracing (MBTT) method to obtain the radiation brightness temperature of rough surface. Hence, the discrimination of brightness temperature tracing method is enhanced.
\end{abstract}

\section{Introduction}

Millimeter-wave (MMW) imaging has been a hot topic in imaging work in recent years; a desirable advantage of MMW imaging is that millimeter waves penetrate cloud and mist and dust [1-4]. Compared with active millimeter-wave imaging, the passive MMW imaging is more widely used because of its good stealth to detect and discriminate target [5-7].

At present time, the work of passive MMW imaging has been restricted by the imaging distance. Entering twenty-first century, along with the development of advanced technology of instantaneous imaging of large field $[8,9]$, passive MMW remote sensing has also been developed, and it has led to the development of simulation in passive MMW imaging, which is of great value in the study of MMW radiation of various kinds of targets. Then, it is helpful to recognize the radiation characteristics of the target, explain some of the radiation phenomena, find the specific radiation patterns, and judge the measured results. In addition, simulation in MMW imaging is also beneficial to the parameter design of the radiometer system, with which the performance and the situation of the radiometer can be assessed conveniently. Also, it has become an effective supplement and substitute for real measurement, which can save time and cost.

Passive MMW imaging technology firstly appears to be that of Salmon et al. [10] where plastic and metal objects microwave imaging is simulated; however only a few specific objects are modeled in this paper. Then, a general model which is suitable for any simple scene is established in [11], whereas the effect of single reflection is just considered in the model, and the coupling between the targets in the scene is not taken into consideration. Then in order to simulate the more complex scenes, a greater level of sophistication in the model proposed in [12] is developed, in which the interaction between the various brightness temperature targets in the model is counted with radiometric method. However, the interaction in brightness temperature tracing method is counted with ray tracing method, which can adjust the resolution in a certain range to improve the flexibility [13-15]. And then, passive MMW imaging system for remote sensing is developed in [16]. Afterwards, brightness temperature simulation in extremely rough surface imaging is obtained in [13, $16,17]$, in which the surface is approximated by Lambertian approximate law. In previous work, the space meshing ray tracing method is used in the brightness temperature tracing 


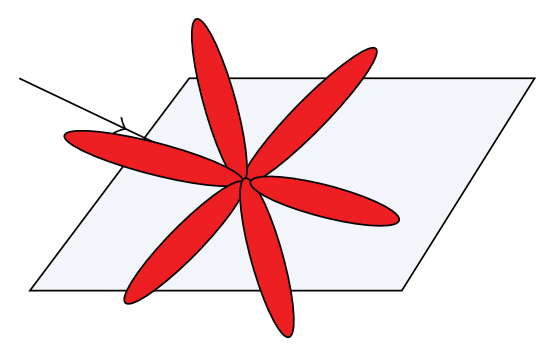

(a)

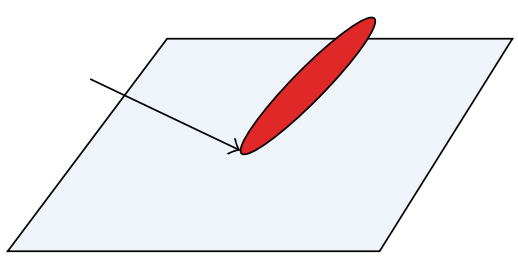

(b)

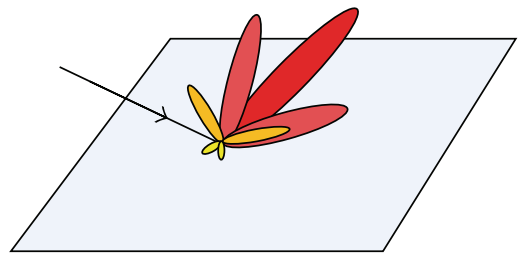

(c)

FIGURE 1: (a) Lambertian model, (b) smooth surface model, and (c) improved model.

which can avoid the intersection test to realize the fast calculation or the highly accurate analysis of the dense ray in the same amount of calculation.

We can reach a conclusion that the scene discussed above is mainly composed of smooth surface or extremely rough surface; however, the simulation in rough surface imaging is not accurate enough because of the approximation in the calculation. To solve the aforementioned problem, we present an improved model in which the diffused reflection of the rough surface is considered and the surrounding brightness temperature in the nonreflection direction is added into the model. Then MBTT method is proposed in this paper to obtain the radiation brightness temperature of rough surface. The results of present work imply that the improved model is closer to the real scene, which effectively enhances the discrimination of different roughness of rough surface.

\section{Model of MBTT Method}

In order to account for diffused reflection in scene simulation, it is important to have a good understanding of the difference between various models. Lambertian approximation law is used in previous work in which the contribution from around brightness temperature to the observation angle is considered as a consistent value. However, only the contribution to the reflection direction should be considered in the model of smooth surface. Actually, the contribution from the brightness temperature around to the brightness temperature in the observation angle is different. To describe the three cases aforementioned clearly, the models are shown in Figures 1(a)$1(\mathrm{c})$.

It can be found that the improved model can be more accurate to account for the diffused reflection of the rough surface, which can improve the discrimination of the brightness temperature tracing method. Then, MBTT method is proposed in this paper to account for the contribution of the diffused reflection of the rough surface.

\subsection{Multilayer Brightness Temperature Tracing (MBTT) Model}

. For the reason that the effect of diffused reflection should be accounted for in the simulation in rough surface imaging, all the rays need to be layered in the tracing process. The principle of layers relies on the times of the diffused reflection of the ray. Rays which do not experience the diffused reflection are considered as the first layer and rays which experience diffused reflection once are considered as the second layer and so on. The schematic diagram is shown in Figure 2.

Different tracing model is applied to different roughness surface; for microrough surface $(0.1<k s<0.3)$, only the brightness temperature in the reflection direction is needed to be considered, whose reflectivity is the addition of rough coefficient based on the reflectivity of smooth surface. For the medium rough surface $(0.3<k s<1)$, reflection brightness temperature is composed of the coherent and incoherent components after diffused reflection; hence, the rays in the reflection direction and those in any other direction are all needed to be considered. Different from medium rough surface, the extremely rough surface is only needed to consider the noncoherent components for extremely rough surface $(k s>1)$, and the coherent components can almost be ignored [16].

The model of medium rough surface is taken as an example to introduce MBTT method for the reason that it includes coherent and incoherent components. The physical model of imaging of space to ground is shown in Figure 3, and the bottom surface is medium rough surface. As shown in Figure 3, the pitch angle and the azimuth angle of radiometer antenna in the coordinate are $(\theta, \varphi)$, which receives the brightness temperature from target 1 , and $T_{\mathrm{AP}}(\theta, \varphi)$ is the brightness temperature arriving at the radiometer which is composed of the air, target 1 , target 2 , and their coupling between each other.

MBTT method uses the receiving point as source point in order to facilitate the calculation. After emitting, if the ray is across a smooth surface, it will continue tracing in the reflection direction, and if the ray is across a rough surface, besides the ray in reflection direction, other rays emitted from the new source are also needed to trace. The new source is the cross point. Then a certain number of times of reflection and layer are set, and then the tracing will be terminated when the times of reflection and layer reach a certain number.

In Figure 4, model of MBTT of a single ray is shown. The radiometer swings in the range of its azimuth angle and pitching angle; each azimuth angle and pitching angle can be considered as a ray, and the interval between the rays can arbitrarily be set if hardware conditions permit. Assume that the range of pitch angle is divided into $o$ segments, and azimuth angle is $p$ segments. In order to distinguish the different reflection and layer in the tracing, brightness temperature of 


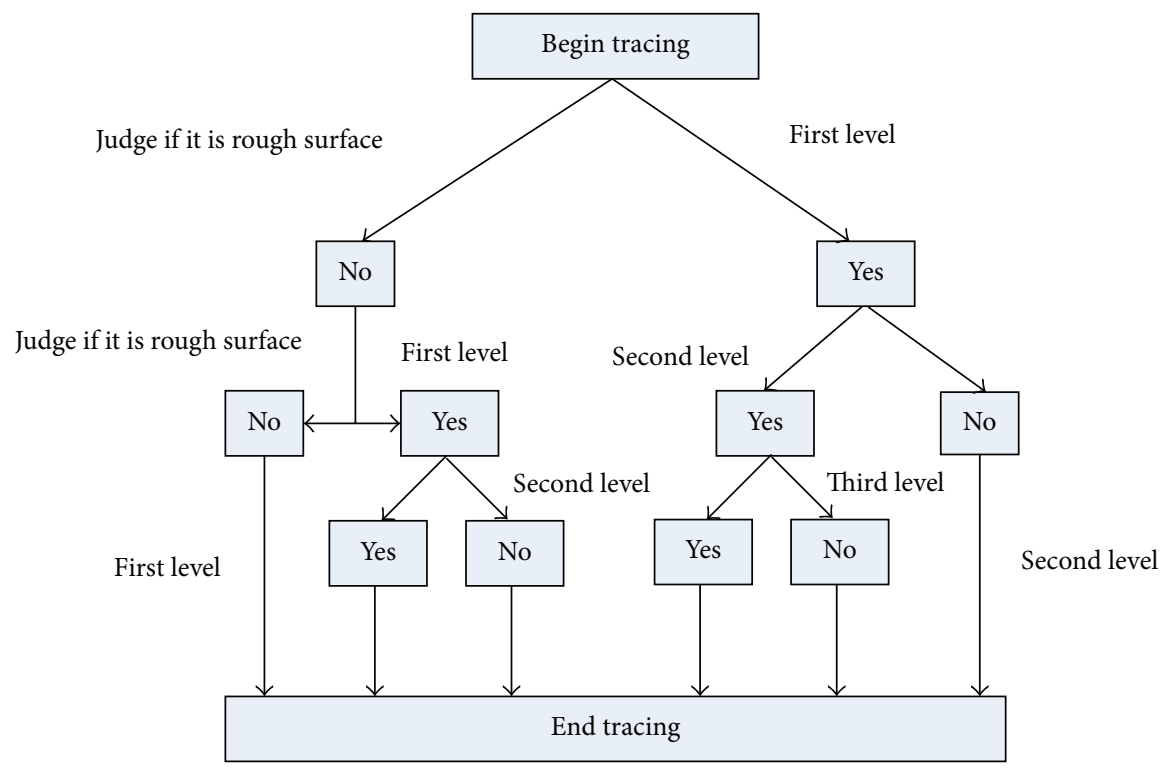

FIGURE 2: Schematic diagram of MBTT method.

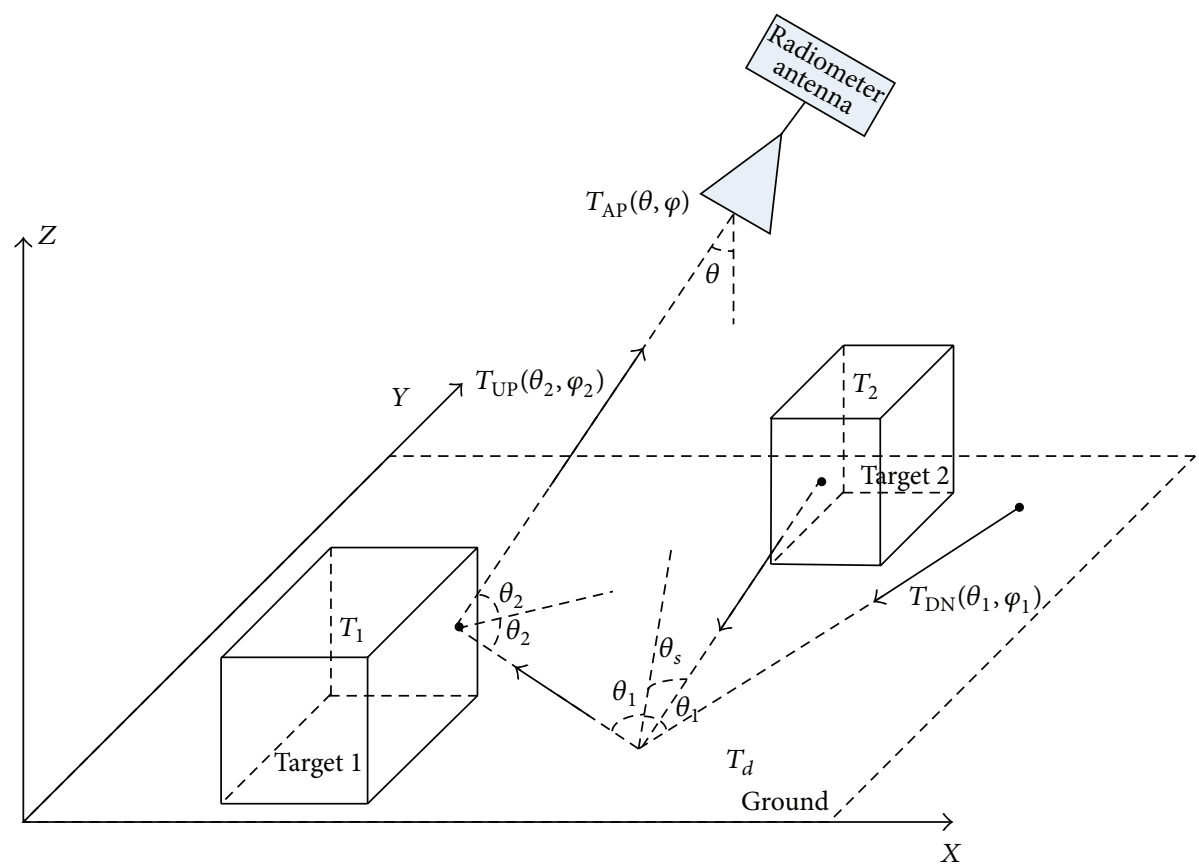

Figure 3: Physical model of space to ground.

each section is represented by $T_{i j}$. The number of layer is expressed by $i$; if the number of maximum layer is set as 3 , then $i=1,3$. The times of reflection are expressed by $j$; if the times of maximum reflection are set as 3 , then $j=$ 0,3 . Therefore, the brightness temperature arriving at the radiometer is represented by $T_{10}\left(\theta_{0}, \varphi_{p}\right)$, and the brightness temperature from the ground to target 1 is expressed by $T_{11}\left(\theta_{2}, \varphi_{2}\right) . \theta_{2}$ is the incident degree of the ray.

After the ray crosses the ground, coherent and noncoherent components will be formatted due to the medium rough surface. The coherent component is the ray in the reflection direction; the brightness temperature from the air to the ground is expressed by $T_{12}\left(\theta_{1}, \varphi_{1}\right)$. And the incoherent component is the ray emitted from the intersection after the ray crosses the rough surface. In the model, the intersection point is regarded as the source of emitting rays, and the second layer tracing begins. As it is shown in Figure 4, $T_{20}\left(\theta_{s}, \varphi_{t}\right)$ represents the brightness temperature from target 2 to the intersection, and $T_{20}\left(\theta_{s+1}, \varphi_{t+1}\right)$ expresses another brightness temperature from the air to the intersection. After 


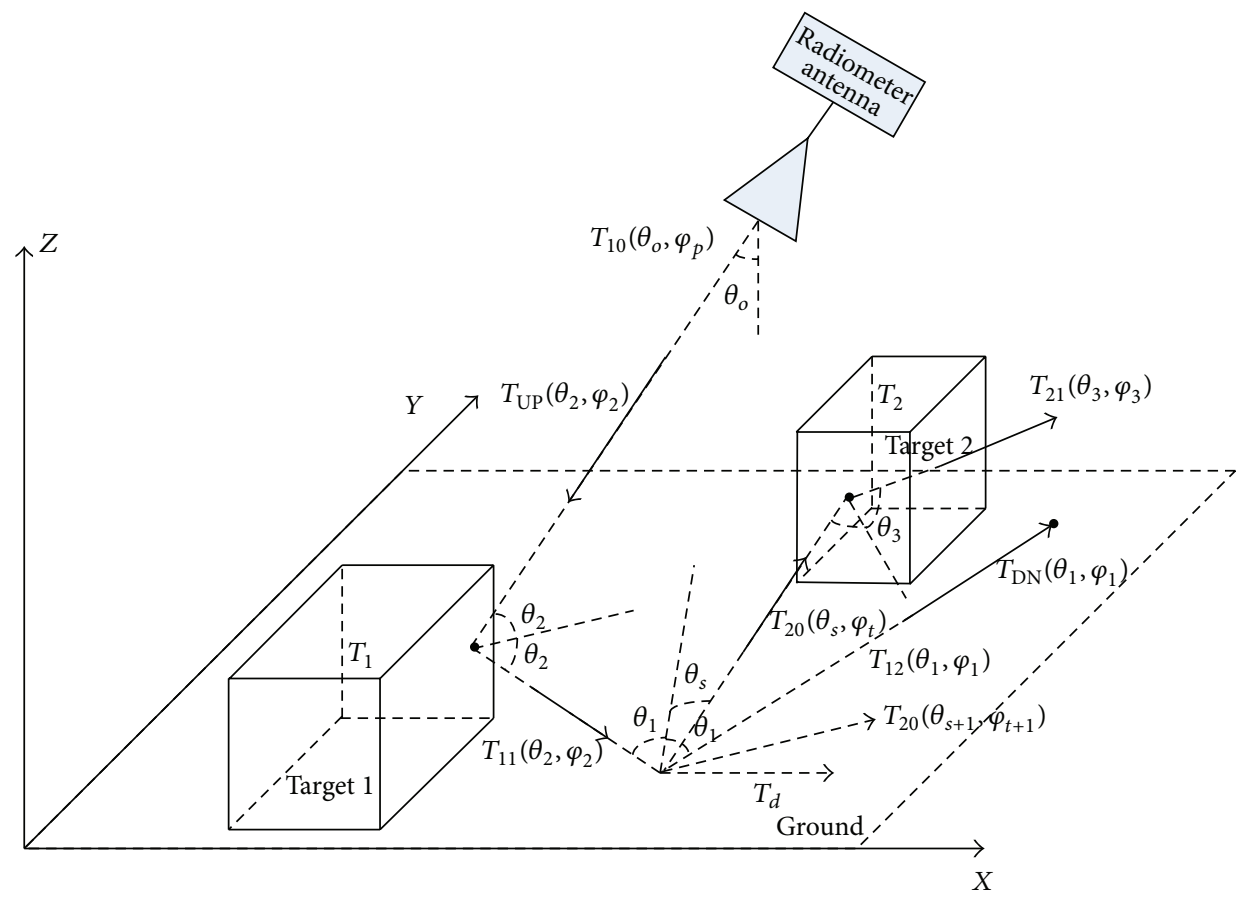

FIGURE 4: Model of MBTT method.

the experiment, it can be concluded that two layers are enough to simulate the radiation brightness temperature of rough surface exactly.

2.2. Retrieving Model of MBTT. For retrieving model, compared with the single layer brightness temperature, besides the layer itself, the coupling between the upper and lower layers also needs to be considered in the MBTT method. As it is shown in Figure 4, $T_{10}\left(\theta_{o}, \varphi_{p}\right)$ represents the brightness temperature arriving at the radiometer, which is composed of the brightness temperature of the air in the incident direction $T_{\mathrm{UP}}\left(\theta_{2}, \varphi_{2}\right)$, the brightness temperature from target $1 T_{1} \cdot e_{1}\left(\theta_{2}\right)$, and the brightness temperature from the ground reflected by target $1 T_{11}\left(\theta_{2}, \varphi_{2}\right) \cdot r_{1}\left(\theta_{2}\right)$, which can be expressed by

$$
\begin{aligned}
T_{10}\left(\theta_{o}, \varphi_{p}\right)= & T_{11}\left(\theta_{2}, \varphi_{2}\right) \cdot r_{1}\left(\theta_{2}\right)+T_{1} \cdot e_{1}\left(\theta_{2}\right) \\
& +T_{\mathrm{UP}}\left(\theta_{2}, \varphi_{2}\right) .
\end{aligned}
$$

For smooth surface, the first two parts in formula (1) are consistent with the general model, and the change is the third item, $T_{11}\left(\theta_{2}, \varphi_{2}\right)$, which represents the brightness temperature arriving at target 1 from the ground. It is composed of brightness temperature in the reflection direction and the ground itself, which is given by formula (2). The polarization rotation coefficient between the rough surface and the reflection surface of target 1 is represented by $D_{12}^{1}$.

$$
\begin{aligned}
T_{11}\left(\theta_{2}, \varphi_{2}\right)= & {\left[T_{12}\left(\theta_{1}, \varphi_{1}\right) \cdot r_{p}\left(\theta_{1}\right)+T_{d} \cdot e_{d p}\left(\theta_{1}\right)\right] } \\
& \cdot D_{12}^{1} .
\end{aligned}
$$

Formula (2) has been obtained in the previous work. And if the Lambertian model is used, $T_{11}\left(\theta_{2}, \varphi_{2}\right)$ in different observation angles will be set as a certain value. However, for modified model, the diffused reflection of the rough surface is accounted for in the model. Hence the modified $T_{11}\left(\theta_{2}, \varphi_{2}\right)$ can be given as follows:

$$
\begin{aligned}
& T_{11}\left(\theta_{2}, \varphi_{2}\right)=\left[T_{12}\left(\theta_{1}, \varphi_{1}\right) \cdot r_{p}\left(\theta_{1}\right) \cdot e^{\left(-4 k^{2} s^{2} \cos ^{2}\left(\theta_{1}\right)\right)}\right. \\
& \left.+T_{2 \text { tol }}\left(\theta_{1}, \varphi_{1}\right)+T_{d} \cdot e_{d p}\left(\theta_{1}\right)\right] \cdot D_{12}^{1}
\end{aligned}
$$

In (3), it can be seen that modified $T_{11}\left(\theta_{2}, \varphi_{2}\right)$ is composed of three parts; the first part is the coherent components, and its reflectance is the addition of rough coefficient based on the reflectance of smooth surface. Then, $T_{12}\left(\theta_{1}, \varphi_{1}\right)$ is actually the brightness temperature of the air down to the ground $T_{\mathrm{DN}}\left(\theta_{1}, \varphi_{1}\right) . r_{p}\left(\theta_{1}\right)$ is the reflectance of smooth surface in direction $\theta_{1}$, while $p$ is the polarization mode and $k$ and $s$ are, respectively, the wave number and RMS height. And then, the second part $T_{2 \text { tol }}\left(\theta_{1}, \varphi_{1}\right)$ is the total brightness temperature of the second layer after diffused reflection in direction $\left(\theta_{1}, \varphi_{1}\right)$ which is the product of the around brightness temperature arriving at the source of the second layer. Using the reflectance $\Gamma\left(\theta_{i}, \varphi_{j} ; \theta_{1}, \varphi_{1}\right)$ from the direction $\left(\theta_{i}, \varphi_{j}\right)$ to the direction $\left(\theta_{1}, \varphi_{1}\right)$, we have

$$
\begin{aligned}
& T_{2 \text { tol }}\left(\theta_{1}, \varphi_{1}\right) \\
& =\sum_{i=1, N} \sum_{j=1, M} T_{20}\left(\theta_{i}, \varphi_{j}\right) \cdot \Gamma\left(\theta_{i}, \varphi_{j} ; \theta_{1}, \varphi_{1}\right),
\end{aligned}
$$

where $T_{20}\left(\theta_{i}, \varphi_{j}\right)$ in (4) is the brightness temperature from arbitrary direction arriving at the source of second layer. In 
Figure 4, brightness temperature $T_{20}\left(\theta_{s}, \varphi_{t}\right)$ is taken as an example, which can be defined by

$$
\begin{aligned}
T_{20}\left(\theta_{s}, \varphi_{t}\right)= & {\left[T_{21}\left(\theta_{3}, \varphi_{3}\right) \cdot r_{2}\left(\theta_{3}\right)+T_{2} \cdot e_{2}\left(\theta_{3}\right)\right] } \\
& \cdot D_{12}^{2}
\end{aligned}
$$

in which $T_{21}\left(\theta_{3}, \varphi_{3}\right)$ is the brightness temperature of the air down to target 2 and $r_{2}\left(\theta_{3}\right)$ and $e_{2}\left(\theta_{3}\right)$ are, respectively, the reflectance and emissivity of target 2 in direction $\theta_{3}$. And $T_{2}$ is the physical temperature of target 2 . The polarization rotation coefficient between the reflection surface of target 2 and the rough surface is represented by $D_{12}^{2}$. In formula (4), the pitch angle direction is divided into $N$ parts, and the azimuth direction is divided into $M$ parts; consequently, $\Gamma\left(\theta_{i}, \varphi_{j} ; \theta_{1}, \varphi_{1}\right)$ is the reflectance from direction $\left(\theta_{i}, \varphi_{j}\right)$ to $\left(\theta_{1}, \varphi_{1}\right)$, which can be obtained by

$$
\Gamma_{p}\left(\theta_{i}, \varphi_{j} ; \theta_{1}, \varphi_{1}\right)=\int_{\theta_{1}-\pi / 2 N}^{\theta_{1}+\pi / 2 N} \int_{\varphi_{1}-\pi / M}^{\varphi_{1}+\pi / M} \frac{\left[\sigma_{p p}\left(\theta_{i}, \varphi_{j} ; \theta_{1}, \varphi_{1}\right)+\sigma_{p q}\left(\theta_{i}, \varphi_{j} ; \theta_{1}, \varphi_{1}\right)\right] \cdot \sin \left(\theta_{1}\right)}{4 \pi \cos \left(\theta_{i}\right)} d \theta_{1} d \varphi_{1}
$$

In (6), $p, q$ are the polarization modes and $\sigma\left(\theta_{i}, \varphi_{j} ; \theta_{1}, \varphi_{1}\right)$ is the reflectance coefficient from direction $\left(\theta_{i}, \varphi_{j}\right)$ to $\left(\theta_{1}, \varphi_{1}\right)$, the derivation of which is contained in [18]. The third part of (1) is the brightness temperature from ground arriving at target 1 , where $T_{d}$ is the physical temperature of the ground and $e_{d p}\left(\theta_{1}\right)$ is the emissivity in $p$ polarization of the ground in direction $\theta_{1} \cdot t_{d p}\left(\theta_{1}\right)$ is the transmittance in $p$ polarization of the ground in direction $\theta_{1}$. The emissivity of smooth surface can be represented by

$$
e_{d p}\left(\theta_{1}\right)=1-r_{d p}\left(\theta_{1}\right)-t_{d p}\left(\theta_{1}\right) .
$$

The contribution of the nonreflection direction should be considered in calculating the emissivity of the rough surface; thus we have

$$
e_{d p}\left(\theta_{1}\right)=1-r_{d p}^{\mathrm{co}}\left(\theta_{1}\right)-r_{d p}^{\mathrm{non}}\left(\theta_{1}\right)-t_{d p}\left(\theta_{1}\right)
$$

in which $r_{d p}^{\mathrm{co}}\left(\theta_{1}\right)$ and $r_{d p}^{\text {non }}\left(\theta_{1}\right)$ are, respectively, its coherent reflection coefficient and noncoherent reflection coefficient. In this case, $t_{d p}\left(\theta_{1}\right)$ in (7) and (8) is 0 . The former has been introduced above, and the latter is given as follows:

$$
\begin{aligned}
& r_{d p}^{\text {non }}\left(\theta_{1}\right) \\
& =\iint_{\Omega} \frac{\left[\sigma_{p p}\left(\theta_{1}, \varphi_{1} ; \theta_{i}, \varphi_{j}\right)+\sigma_{p q}\left(\theta_{1}, \varphi_{1} ; \theta_{i}, \varphi_{j}\right)\right] \cdot \sin \left(\theta_{i}\right)}{4 \pi \cos \left(\theta_{1}\right)} d \theta_{i} d \varphi_{j} .
\end{aligned}
$$

We can obtain the modified brightness temperature from the ground to target $1 T_{11}\left(\theta_{2}, \varphi_{2}\right)$ by combining (4), (5), (6), (8), and (9) with (3); after that, the retrieving can proceed according to the way of general brightness temperature tracing, and the final brightness temperature arriving at the radiometer can be obtained.

\section{Experiments and Analysis}

3.1. Comparing with the Grass. To verify the model discussed before, we measure the brightness temperature of grass by use of radiometer antenna. Figure 5 shows the grass scene measured using a millimeter radiometer.

The results are shown in Figure 6, and the calculated results using the improved model are also shown in Figure 6. The working frequency is $94 \mathrm{GHz}$, and the root mean square height and the correlation length are $3.5 \lambda$ and $10 \lambda$, respectively. The results reveal that the value obtained by the improved model is closer to the measured value; however the value of Lambertian model is significantly different from the measured one. Therefore, it may not be able to meet the requirement of detection in some specific condition.

We can conclude from the above that Figure 6 demonstrates the correctness and feasibility of the improved model. The relations between the simulation time with the number of layers and the times of reflection for the scene in Figure 5 are shown in Figure 7.

In Figure 7(a), the simulation time increases with the layer number of the model, and, in Figure 7(b), the simulation time increases almost linearly with the reflection times. This is because the transmitting ray number is constant and the ray lengths increase with reflection times.

Actually the relative error of brightness temperature decreases with the layer number, which will approach a constant value. In the simulation for single layer model, the relative error of the results mainly comes from the inexact modeling of the rough surface, which can be obviously decreased by increasing the layer number. However, if the error caused by inexactness of the rough surface is decreased with several layers, the errors from other inexactness of the details of the scene, such as media and background parameters, measured values will become the dominant part. Then, the simulation accuracy cannot be improved by only increasing the layer and the relative error tends to be a constant value. This can be seen in Figure 8, in which the relative errors are calculated by comparison with the measured value shown in Figure 6.

To further study the effect of improved model, the simulation in three-dimensional scene imaging is presented in the following.

\subsection{Simulations in MMW Imaging of Plane Runway. Figures} 9(a) and 9(b) are, respectively, the optical and measured images of airplane runway. It can be seen in the optical image that the rough surface is the grass next to the runway, which is at least composed of two different lengths of grass. Different brightness represents various grass, which is already marked in the figures. In the calculation, the length of the long grass and short grass is, respectively, set as $20 \lambda$ and $3.5 \lambda$. Hence, in 


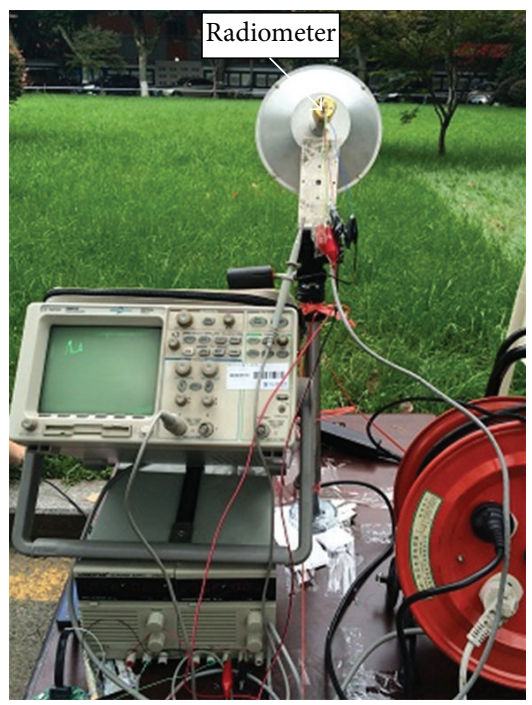

FIGURE 5: Measured scene of the grass.

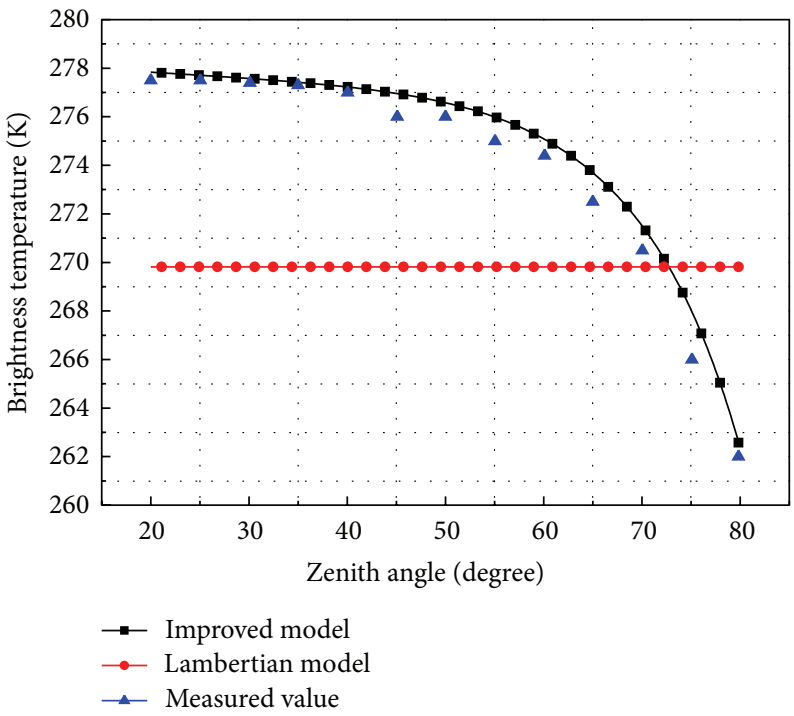

Figure 6: Comparison of the three models.

the measured image, it is easy to discriminate the two kinds of different roughness of the grass due to the different colors.

The simulated image of runway is, respectively, obtained in Figures 10 and 11, in which the two different models are applied. It appears clearly that both of the images reproduce the measured image of the radiometer well; however, a slight difference between the measured image and simulated image is due to the modeling of the scene. Figure 10 indicates that the brightness temperature of the grass is exactly the same for different roughness of grass. However, the color of the different roughness has a significant difference in Figure 11, which is similar to the measured image.

To facilitate observation, the brightness temperature of the short grass is appropriately treated in this paper, which is shown in Figure 12. Thus, the effectiveness and advantage of the improved model is further verified by comparing the measured image.

3.3. Simulations in $M M W$ of Virtual Case. To study the simulation in different roughness of rough surfaces imaging, we present a three-dimensional virtual scene in this paper, in which the improved model and the original model are used. The model of the scene is shown in Figure 13, which includes four planes, respectively, 76, 79, 82, and 85, located on the $x-O-y$ plane. In addition, the four rectangular bodies are located on the four planes, and the radiometer is located at the top of the scene. The coordinates of the radiometer are $(0$, $0,20)$, and the origin of the coordinate system is located on the $x-o-y$ plane under the radiometer. The center direction of radiometer is $\left(\theta=130^{\circ}, \varphi=0^{\circ}\right)$, and the deviation on 


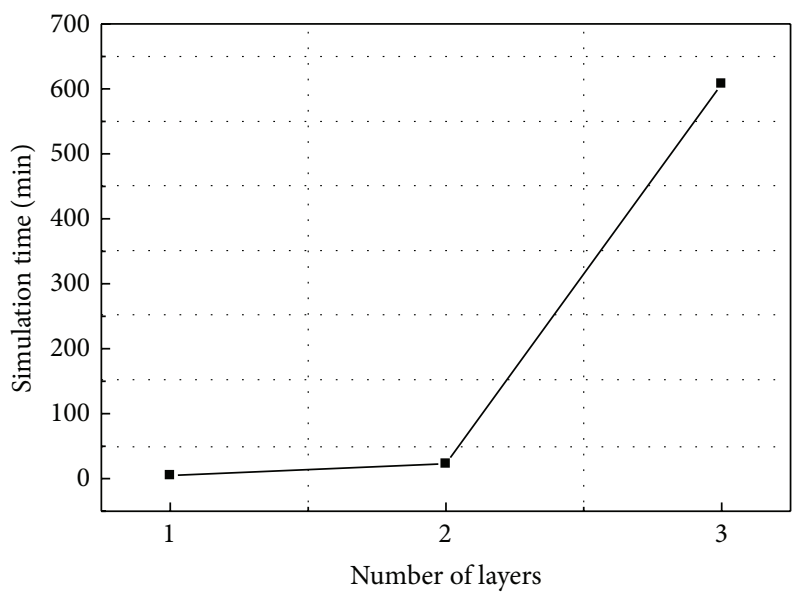

(a)

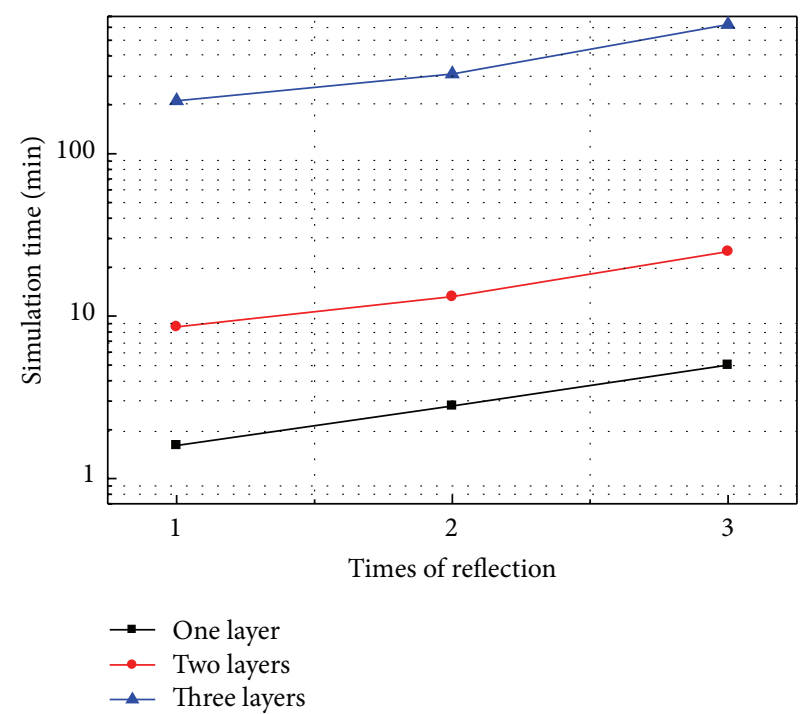

(b)

FIGURE 7: (a) Simulation time with the layers considered in the model. (b) Simulation time with the times of reflection considered in the model.

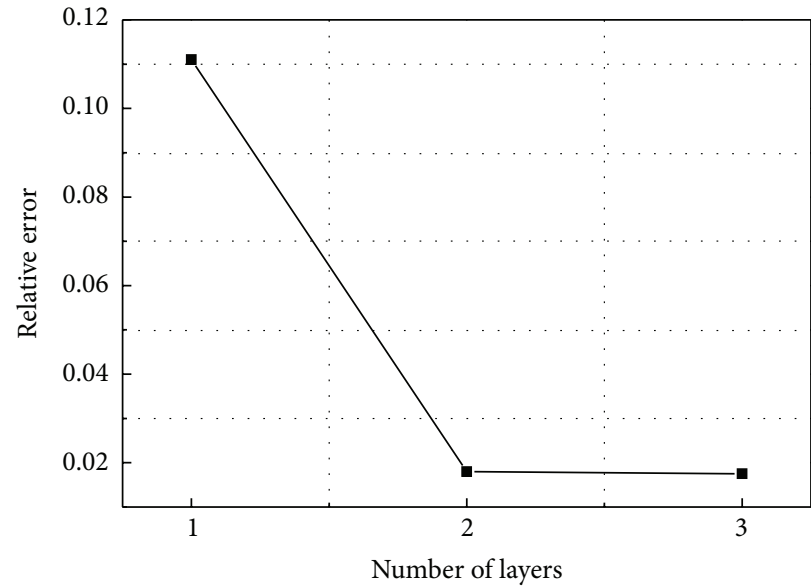

FIGURE 8: Relative errors with the number of layers.

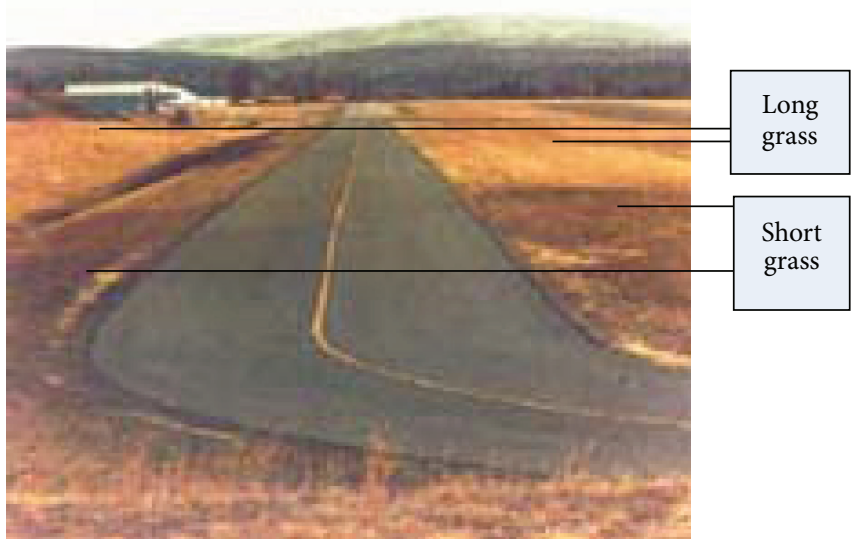

(a)

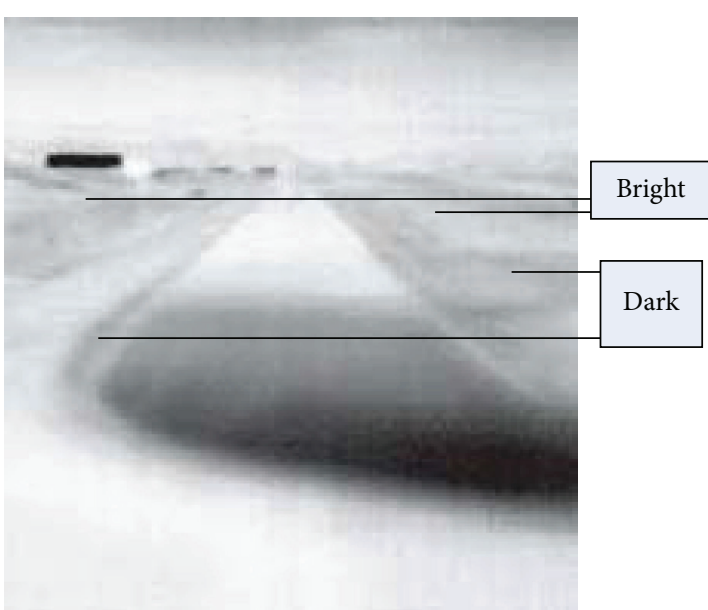

(b)

FIGURE 9: Optical image and measured image of airplane runway. 


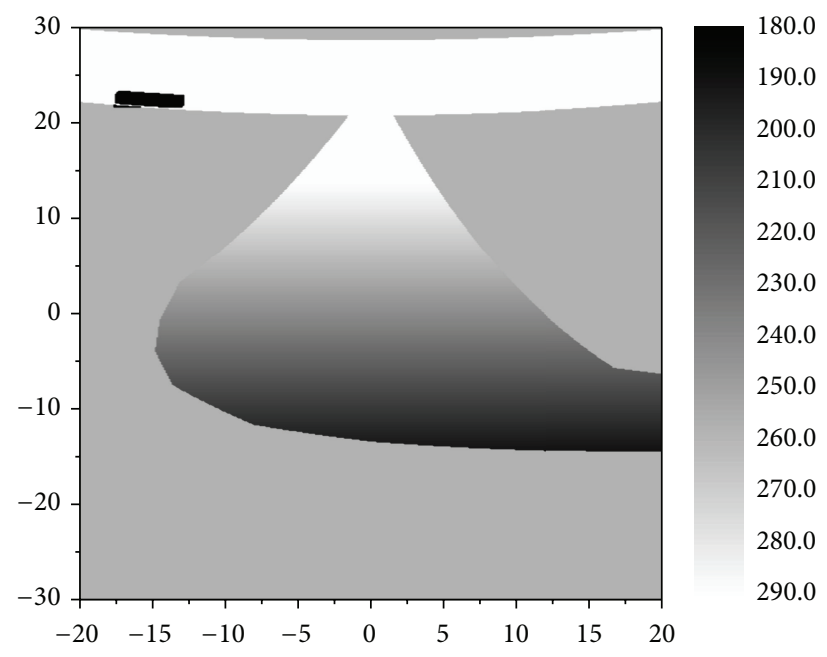

FIgURE 10: Simulated image of Lambertian model.

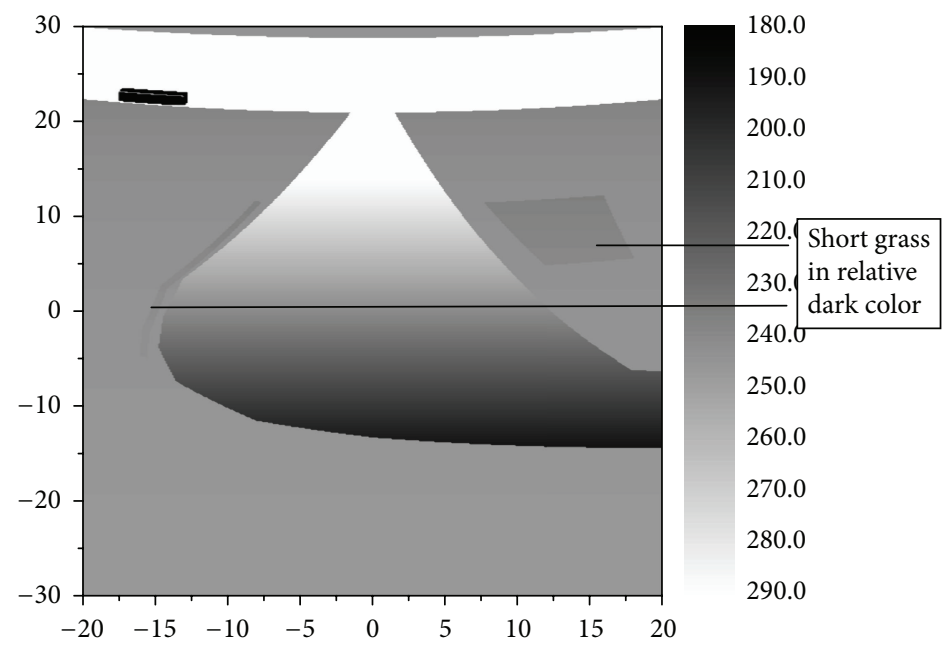

Figure 11: Simulated image of improved model.

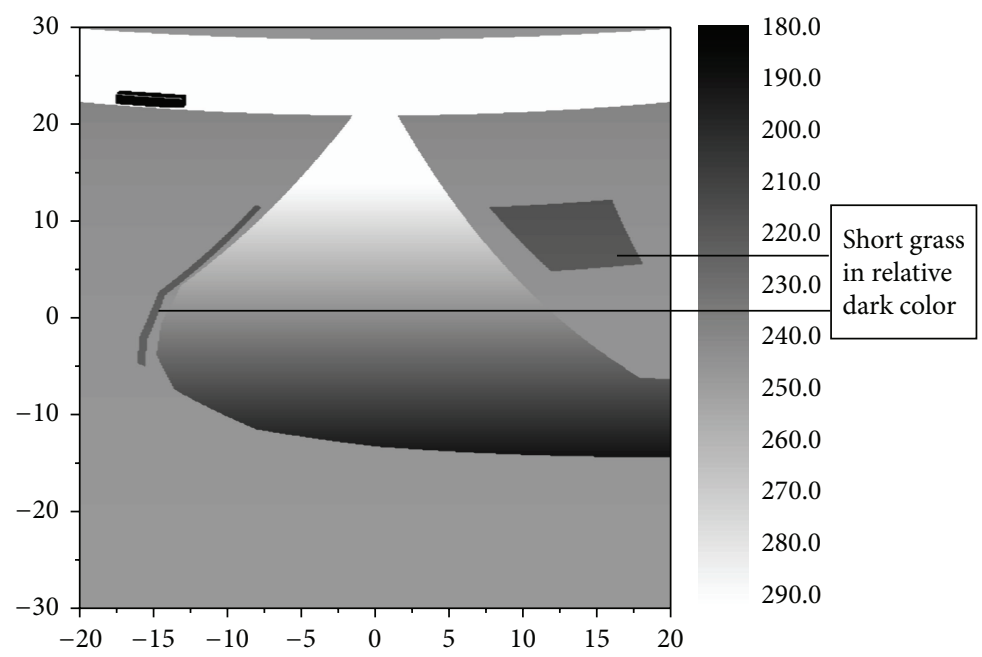

FIGURE 12: Revised simulated image of improved model. 


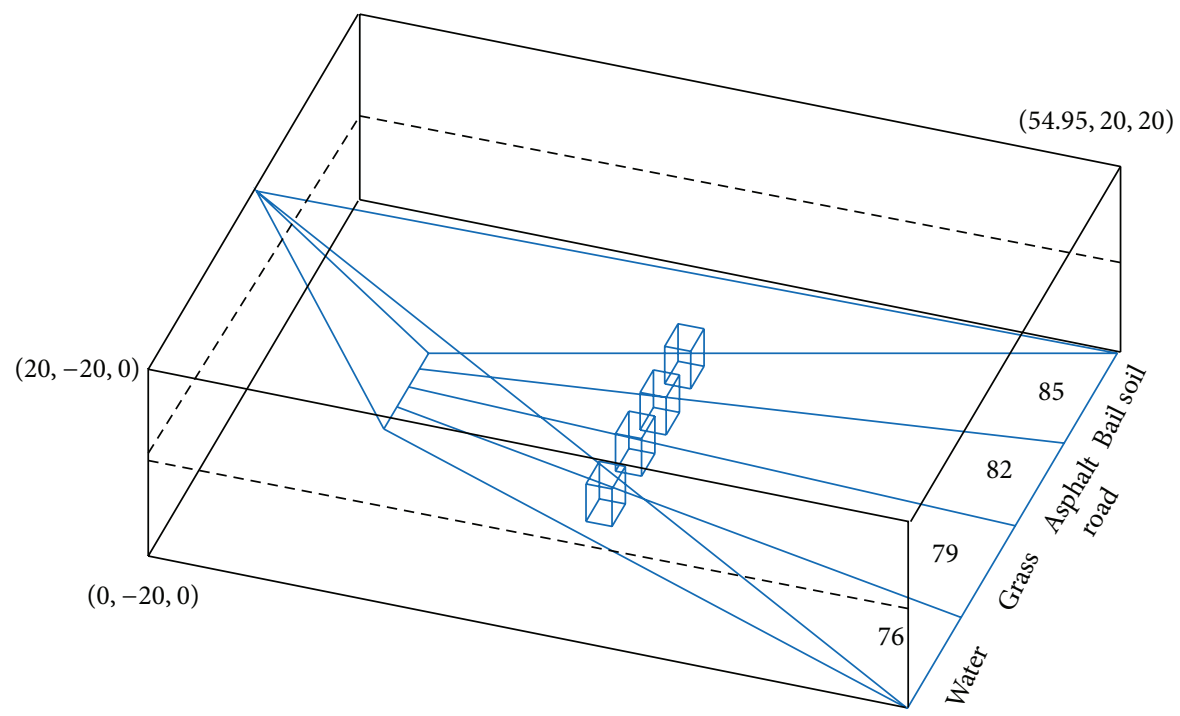

FIGURE 13: Virtual scene of rough surfaces of different roughness, in which the working frequency is assumed to be $94 \mathrm{GHz}$ and the relative complex permittivity of the four surfaces is assumed to be $(81.0,1.0),(1.353,0.070),(6.0,0.1)$, and $(2.1,0.9)$, consequently, and the relative complex permittivity of the four rectangular bodies is assumed to be $(3.50,1.20)$ which is like the cement material. In the scene, the average brightness temperature of sky background, rectangles, and planes is assumed to be $50 \mathrm{~K}, 300 \mathrm{~K}$, and $200 \mathrm{~K}$, respectively.

the azimuth angle and pitching angle is both $40^{\circ}$. Four planes from 76 to 85 are assumed to be water, grass, asphalt road, and bare soil in turn. The four planes can be considered as four different roughness surfaces; the asphalt is considered as a microrough surface, the water surface is considered as a medium rough surface, and the bare soil and grass are considered as an extremely rough surface. It must be pointed out that in this paper the ambient temperature of the target is not taken into account by the environmental factors such as rainfall, fog, and cloud cover [19-22].

Figure 14(a) is the simulated image in which the original model is used; Lambertian model is used for grass and soil. The slightly rough surface and medium rough surface are not mentioned in previous paper; hence, the emissivity of the two kinds of rough surface is also set as a certain value for convenient comparison of the data.

Figure 14(c) is the simulated image, which uses the improved model proposed in this paper where the roughness of the surface is described by RMS height $\sigma$ and correlation length $L$. The four rectangles can be clearly seen in both of the images, and the brightness temperature of grass, bare soil, asphalt, and water is decreasing.

The difference between Figures 14(a) and 14(c) is that the brightness temperature of the four rough surfaces is all the same in deviation angle from $-20^{\circ}$ to $+20^{\circ}$ in Figure 14(a). However, the brightness temperature of four rough surfaces is changing with the variation of pitching angle in Figure 14(c). Moreover, brightness temperature of asphalt and water is increasing with the elevation of zenith angle; however grass and bare soil have the converse results.

In order to observe the change of brightness temperature with pitch angle of each kind of material, six curves are calculated when the azimuth angle is $-12^{\circ}, 2^{\circ}$, and $12^{\circ}$ in Figures 14(a) and 14(c), which is presented in Figure 14(b).
This study is based on the fact that the brightness temperature is related to the pitch angle but not the azimuth angle. The results indicate that the difference between the two kinds of models is more obvious in Figure 14(b).

3.4. Simulations in MMW of Different Roughness Material. To research the change of brightness temperature with the incident angle in different roughness, Figure 15 reveals the curves of different RMS heights of the same material. The results show that the brightness temperature of the grass increases with the increment of the RMS height. And Figure 16 reveals the curves of different correlation lengths of the same material. Then, the results indicate that the brightness temperature of the grass decreases with the increase of the correlation length. Therefore, it can be concluded that the roughness and the brightness temperature are increased with the roughness in the same medium for the reason that both the increment of RMS height and the decrements of correlative length mean the increment of roughness.

The simulation in different roughness grass imaging is shown in Figure 17. The result indicates that the rangeability of brightness temperature with incident angle also increases with the increment of roughness. It can be seen clearly that the change of brightness temperature of Figure 17(a) is obvious from the top to the bottom, which cannot be observed clearly in Figure 17(d). Therefore, it can be concluded that the difference of brightness temperature between the two models increases with the decrease of roughness, which confirms the effect of the improved model proposed in this paper.

The soil of different humidity is simulated to study the change of brightness temperature of different medium with the incident angle, in which the dielectric constant of soil with different moisture refers to $[23,24]$. The results in 


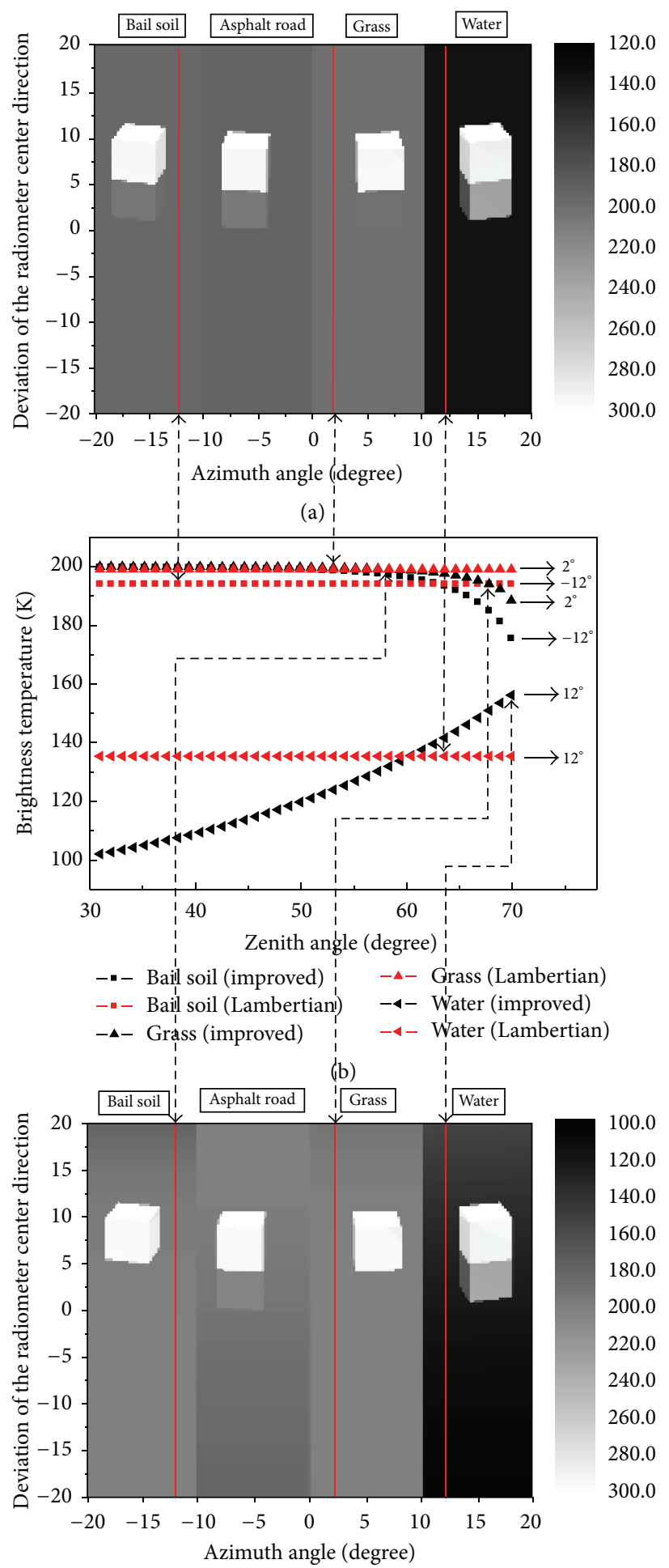

(c)

FIGURE 14: (a) Simulated image of Lambertian model. (b) Comparison between improved models with the Lambertian model. (c) Simulated image of improved model. In (a) and (c) the parameters of the four rough surfaces from left to right are, respectively, $(\sigma=0.0003 \mathrm{~m}, L=1.6 \lambda)$, $(\sigma=0.15 \mathrm{~m}, L=10 \lambda),(\sigma=0.0001 \mathrm{~m}, L=1.6 \lambda)$, and $(\sigma=0.1 \mathrm{~m}, L=10 \lambda)$. 


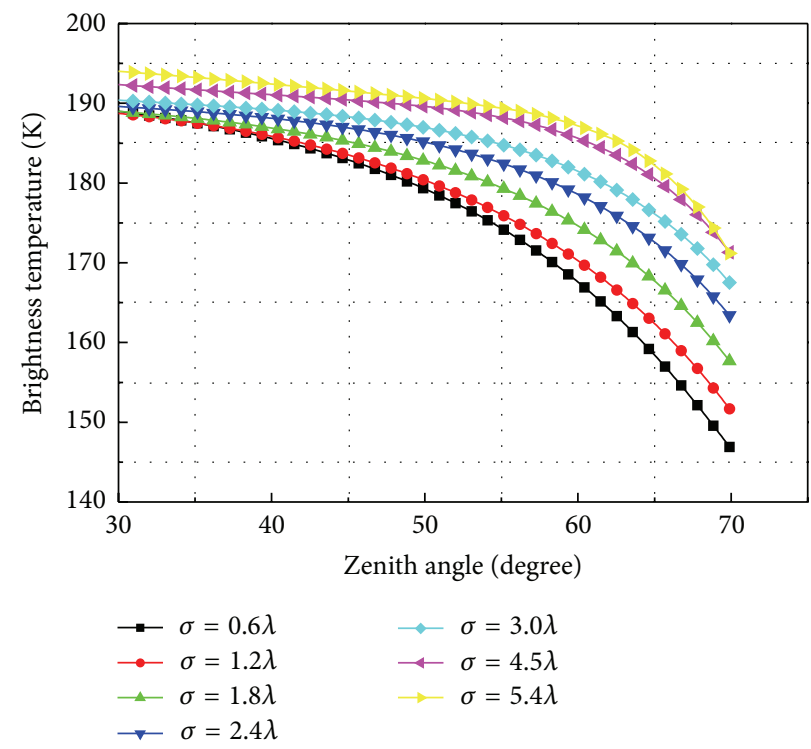

FIGURE 15: Comparison of different RMS heights, in which the correlative length is set as $10 \lambda$ and the relative complex permittivity is set as $(3.2,0.01)$.

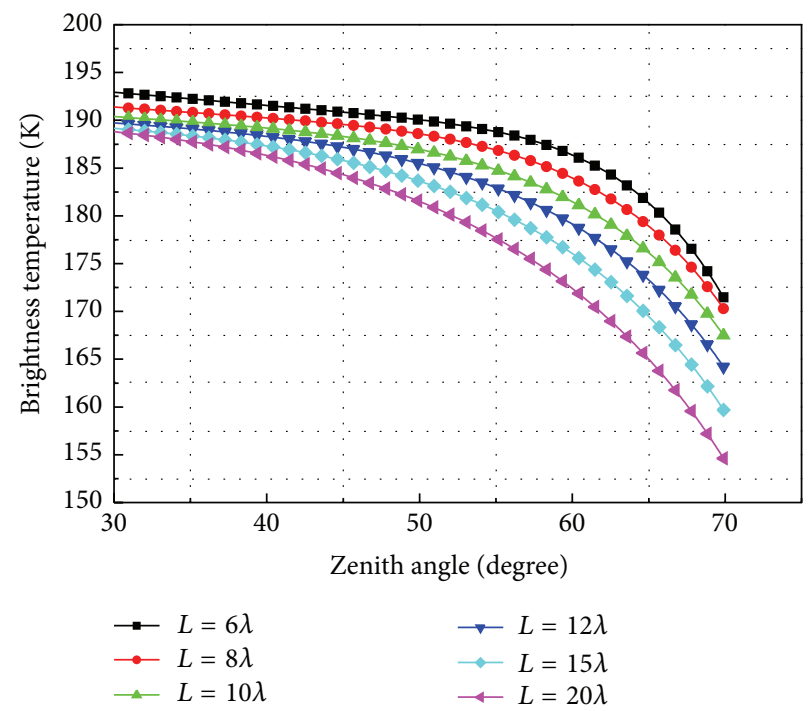

FIGURE 16: Comparison of different correlative lengths, in which the RMS height is set as $3 \lambda$ and the relative complex permittivity is set as $(3.2,0.01)$.

Figures 18 and 19 reveal that the relative permittivity of wet soil is proportional to the content of water, and the brightness temperature decreases with the increment of the relative complex permittivity. The result is consistent with the description of Figures 14(a) and 14(c). Also, it can be concluded that in Figures 18 and 19, for the same medium, the brightness temperature increases with the roughness which is determined by the RMS height and the correlation length. And this conclusion is consistent with the idea described before. In addition, the effect of roughness on the brightness temperature is less than that of the dielectric constant.

\section{Conclusions}

Improved model is proposed in this paper for rough surface scene simulation in millimeter-wave imaging. And MBTT method is suggested to consider the diffused reflection of rough surface. Our results indicate that the improved model is correct and effective in the simulation of the rough surface, whereas the discrimination of the brightness temperature tracing method is improved, with which different roughness of the same material can be clearly discriminated.

In addition, the effects of different root mean square height, correlation length, and the roughness of the dielectric 

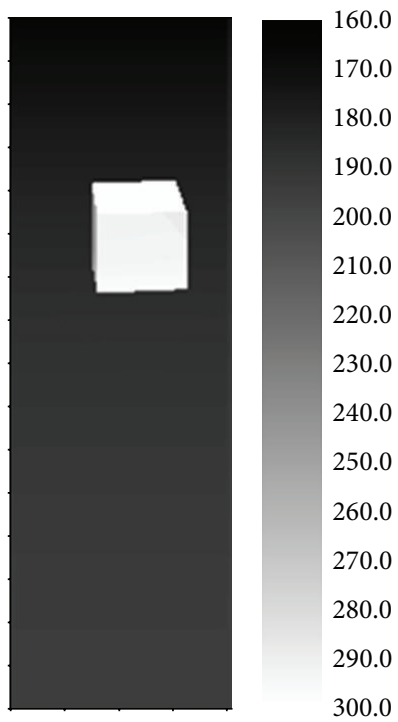

(a) $(\sigma=1.8 \lambda)$

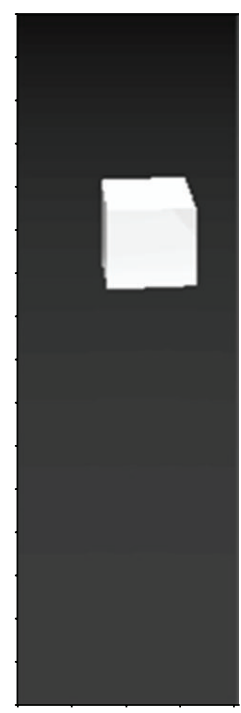

(b) $(\sigma=2.4 \lambda)$

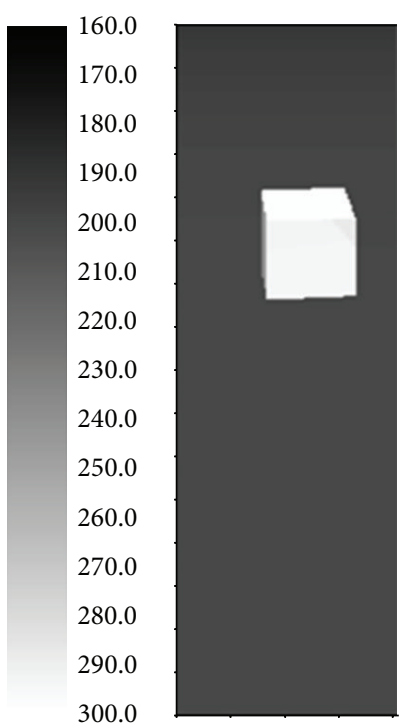

(c) $(\sigma=3.0 \lambda)$

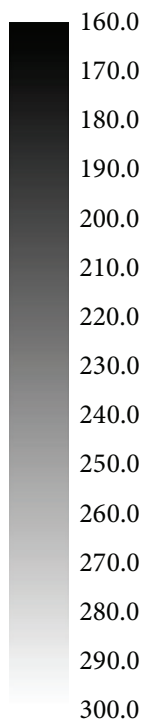

00.0

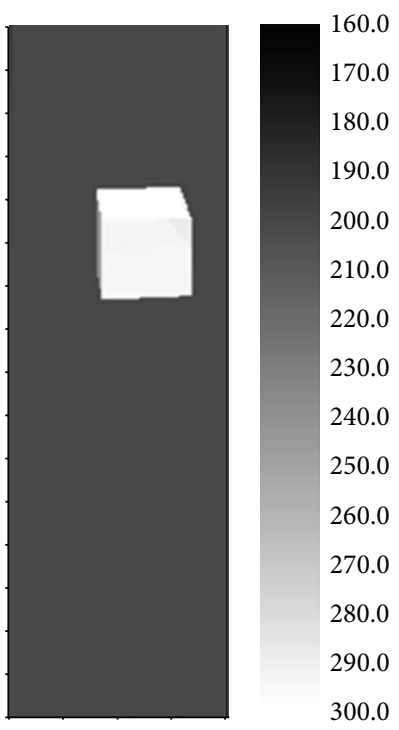

(d) $(\sigma=4.5 \lambda)$

FIGURE 17: Simulated image of brightness temperature of four different types of grass roughness, in which the correlation length of $L$ is $10 \lambda$; from (a) to (d), the RMS height of the rough surface is $1.8 \lambda, 2.4 \lambda, 3 \lambda$, and $4.5 \lambda$, respectively, which means that the roughness from (a) to (d) increases.

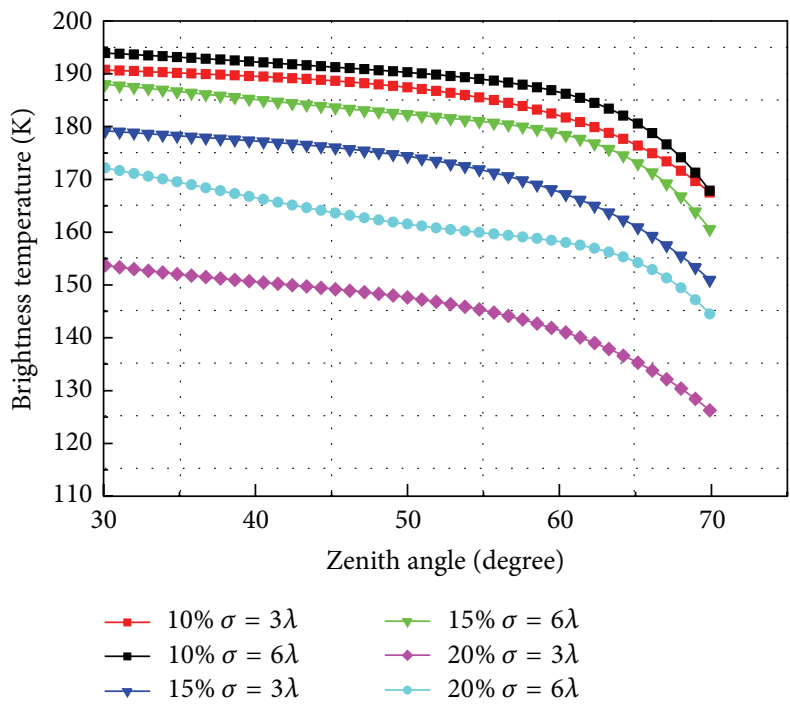

FIGURE 18: Comparison of different RMS heights of wet soil with different moisture content, in which the correlation length of $L$ is $10 \lambda$, and the relative complex permittivity of $10 \%$ humidity, $15 \%$ humidity, and $20 \%$ humidity is set as 2.1-j0.9, 3.0-j1.23, and 1.73-j3.0.

constant of the rough surface are studied and analyzed. The results show that, for the same material, the radiation brightness temperature increases with the increment of the root mean square height; however the correlation length is inversely proportional to the radiation brightness temperature. Hence, it can be concluded that the roughness increases with the increase of the brightness temperature in the same medium for the reason that both the increment of RMS height and the decrements of correlative length mean the increment

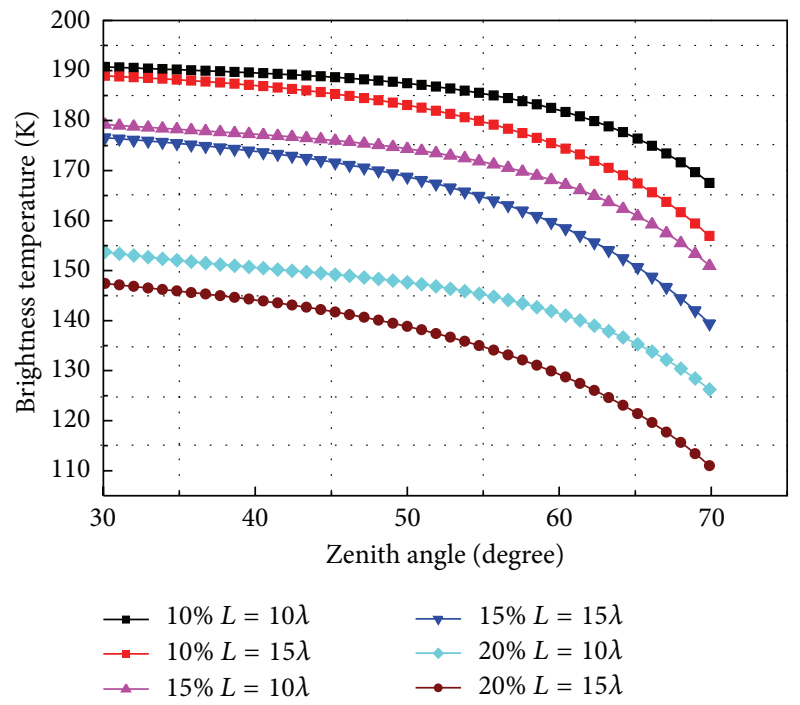

FIGURE 19: Comparison of different correlation lengths of wet soil with different moisture content, in which the root mean square height is $3 \lambda$, and the relative complex permittivity of $10 \%$ humidity, $15 \%$ humidity, and $20 \%$ humidity is set as $2.1-j 0.9,3.0-j 1.23$, and 1.73 j3.0.

of roughness. Besides, the results in this paper reveal that the rangeability of brightness temperature with incident angle increases with the increment of roughness as well. And the radiant brightness temperature decreases with the increment of the dielectric constant. Of course, MBTT method used in this paper is not absolutely perfect, in which the Kirchhoff approximation method is used; however a better method can be applied to calculate the scattering coefficient of rough surface, which will be argued in the future work. 


\section{Competing Interests}

The authors declare that they have no competing interests.

\section{Authors' Contributions}

Chuan Yin was the main author of the manuscript, conceived the study with coauthors, supervised the data analysis, and edited the manuscript. Ming Zhang and Yaming Bo revised the manuscript.

\section{Acknowledgments}

This work is supported by the National Natural Science Foundation of China State Key Laboratory of Millimeter Waves, Natural Science Foundation of Jiangsu Province, and Specialized Research Fund for the Doctoral Program of Higher Education under Grant nos. 61071021, K201413, BK2012435, and 20133223120005, respectively.

\section{References}

[1] G.-D. Liu and Y.-R. Zhang, "Three-dimensional microwaveinduced thermo-acousticimaging for breast cancer detection," Acta Physica Sinica, vol. 60, no. 7, Article ID 074303, pp. 1-7, 2011.

[2] W.-J. Ji and C.-M. Tong, "Research on electromagnetic scattering computation and synthetic aperture radar imaging of ship located on two-dimensional ocean surface," Acta Physica Sinica, vol. 61, no. 16, Article ID 160301, pp. 1-8, 2012.

[3] K. R. Dai, G. X. Liu, Z. H. Li et al., "Extracting vertical displacement rates in Shanghai (China) with multi-platform SAR images," Remote Sensing, vol. 7, no. 8, pp. 9542-9562, 2015.

[4] A. Gorrab, M. Zribi, N. Baghdadi, B. Mougenot, P. Fanise, and Z. L. Chabaane, "Retrieval of both soil moisture and texture using TerraSAR-X images," Remote Sensing, vol. 7, no. 8, pp. 1009810116, 2015.

[5] G.-F. Zhang, X.-G. Li, and G.-W. Lou, "Research on passive MMW imaging based on an alternating current radiometer," Journal of Infrared and Millimeter Waves, vol. 26, no. 6, pp. 461464, 2007.

[6] Y.-D. Zhang, Y.-S. Jiang, Y.-T. He, and H.-Y. Wang, "Passive millimeter-wave imaging using photonic processing technology," Journal of Infrared and Millimeter Waves, vol. 30, no. 6, pp. 551-555, 2011.

[7] S. I. Khan, Y. Hong, J. J. Gourley, M. U. Khattak, and T. De Groeve, "Multi-sensor imaging and space-ground crossvalidation for 2010 flood along Indus River, Pakistan," Remote Sensing, vol. 6, no. 3, pp. 2393-2407, 2014.

[8] C. S. Ruf, C. T. Swift, A. B. Tanner, and D. M. Le Vine, "Interferometric synthetic aperture microwave radiometry for the remote sensing of the earth," IEEE Transactions on Geoscience and Remote Sensing, vol. 26, no. 5, pp. 597-611, 1988.

[9] L. Yujiri, "Passive millimeter wave imaging," in Proceedings of the IEEE MTT-S International Microwave Symposium Digest, vol. 4, pp. 98-101, IEEE, San Francisco, Calif, USA, June 2006.

[10] N. A. Salmon, R. Appleby, and S. Price, "Scene simulation of passive millimeter-wave images of plastic and metal objects," in Proceedings of the Infrared and Passive Millimeter-wave Imaging Systems: Design, Analysis, Modeling, and Testing, vol. 4719 of Proceedings of SPIE, pp. 397-401, Orlando, Fla, USA, July 2002.
[11] N. A. Salmon, "Polarimetric scene simulation in millimeterwave radiometric imaging," in Radar Sensor Technology VIII and Passive Millimeter-Wave Imaging Technology VII, vol. 5410 of Proceedings of SPIE, pp. 260-269, Orlando, Fla, USA, April 2004.

[12] N. A. Salmon, "Polarimetric passive millimetre-wave imaging scene simulation including multiple reflections of subjects and their backgrounds," in Technologies for Optical Countermeasures II; Femtosecond Phenomena II; and Passive Millimetre-Wave and Terahertz Imaging II, Proceedings of SPIE, pp. 354-358, November 2005.

[13] C. Zhang and J. Wu, "Near-field 3D scene simulation for passive microwave imaging," in Proceedings of the Geoinformatics: Remotely Sensed Data and Information, vol. 6419 of Proceedings of SPIE, pp. 1-11, Wuhan, China, October 2006.

[14] C. Zhang and J. Wu, "Image simulation for ground objects microwave radiation," Journal of Electronics \& Information Technology, vol. 29, no. 11, pp. 2425-2728, 2007.

[15] M. R. Fetterman, J. Doughertys, and W. L. Kiser Jr., "Scene simulation of millimeter-wave images," in Proceedings of the IEEE Antennas and Propagation Society International Symposium (AP-S '07), pp. 1493-1496, IEEE, Honolulu, Hawaii, USA, June 2007.

[16] M. R. Fetterman, J. Grata, G. Jubic, W. L. Kiser Jr., and A. Visnansky, "Simulation, acquisition and analysis of passive millimeter-wave images in remote sensing applications," Optics Express, vol. 16, no. 25, pp. 20503-20515, 2008.

[17] N. A. Salmon, "Scattering in polarimetric millimetre-wave imaging scene simulation," Proceedings of SPIE, vol. 6211, pp. 7178, 2006.

[18] F. T. Ulaby, R. K. Moore, and A. K. Fung, Microwave Remote Sensing: Active and Passive, Addison-Wesley, 1982.

[19] H. J. Liebe, G. A. Hufford, and M. G. Cotton, "Propagation modeling of moist air and suspended water/ice particles at frequencies below $1000 \mathrm{GHz}$," in AGARD, Atmospheric Propagation Effects Through Natural and Man-Made Obscurants for Visible to MM-Wave Radiation, SEE N94-30495 08-32, p. 11, 1993.

[20] ITU Recommendation, "Attenuation by atmospheric gases," P.676-5, 200122 ITU-R Recommendation, 'Reference standard atmospheres', P.835-3, 1999.

[21] ITU Recommendation, "Attenuation due to clouds and fog," pp. 840-843, 1999.

[22] ITU, "Reference standard atmospheres," ITU Recommendation 835-3, 1999.

[23] J. R. Zhang, D. H. Zhang, L. W. Wang, Y. Z. Zhao, W. Sheng, and W. Guo, "In situ measurement of typical objects' permittivities in microwave remote sensing," Journal of Electronics, vol. 4, pp. 566-569, 1997.

[24] J. R. Zhang, "The microwave dielectric constant of canopy and soil," Remote Sensing Technology and Application, vol. 10, pp. 4050, 1995. 


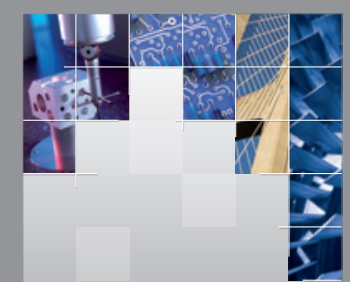

\section{Enfincering}
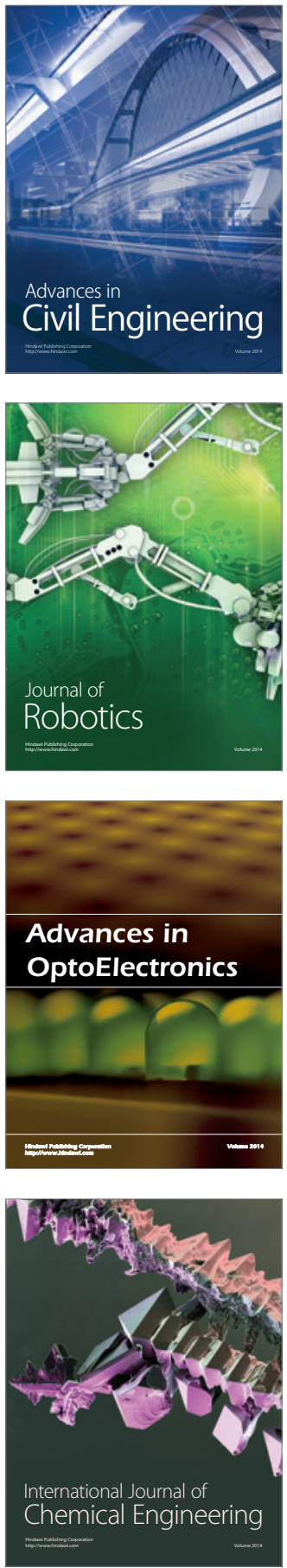

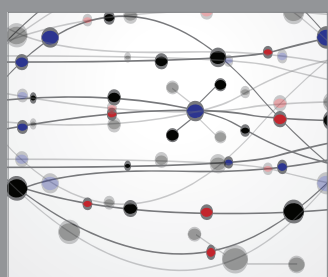

The Scientific World Journal

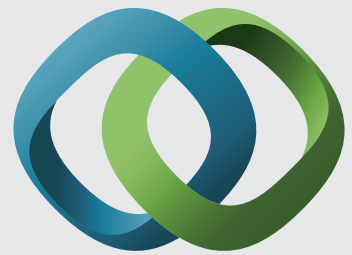

\section{Hindawi}

Submit your manuscripts at

http://www.hindawi.com
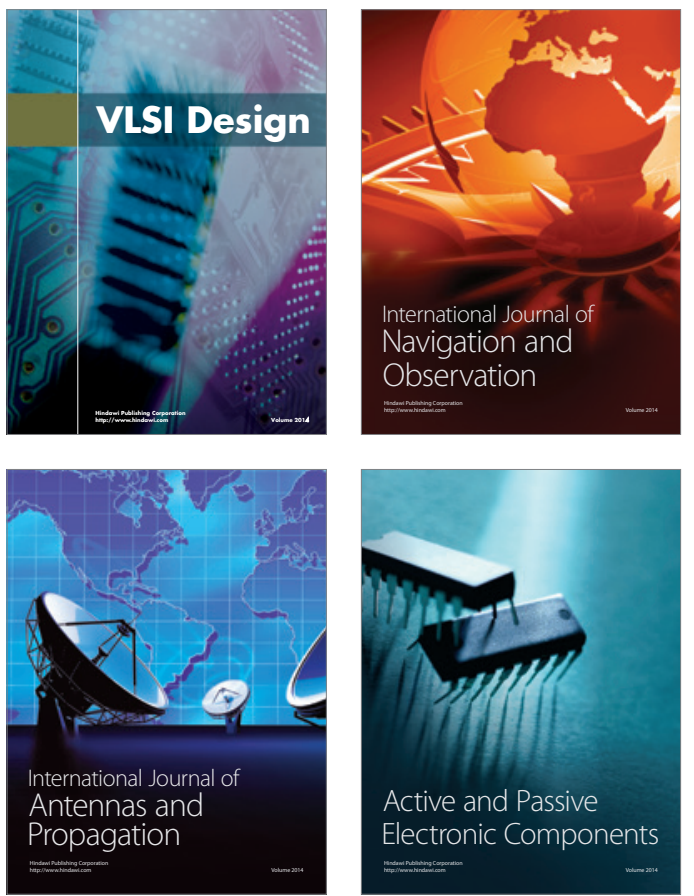
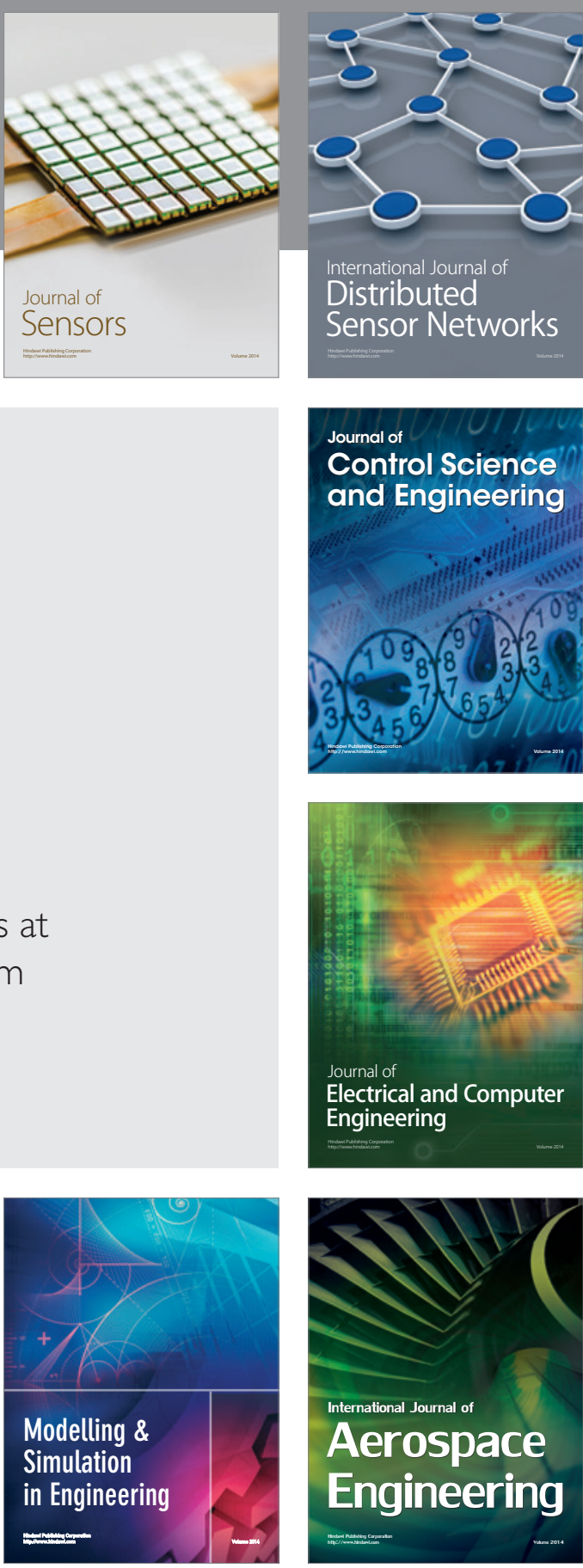

International Journal of

Distributed

Sensor Networks

Journal of

Control Science

and Engineering
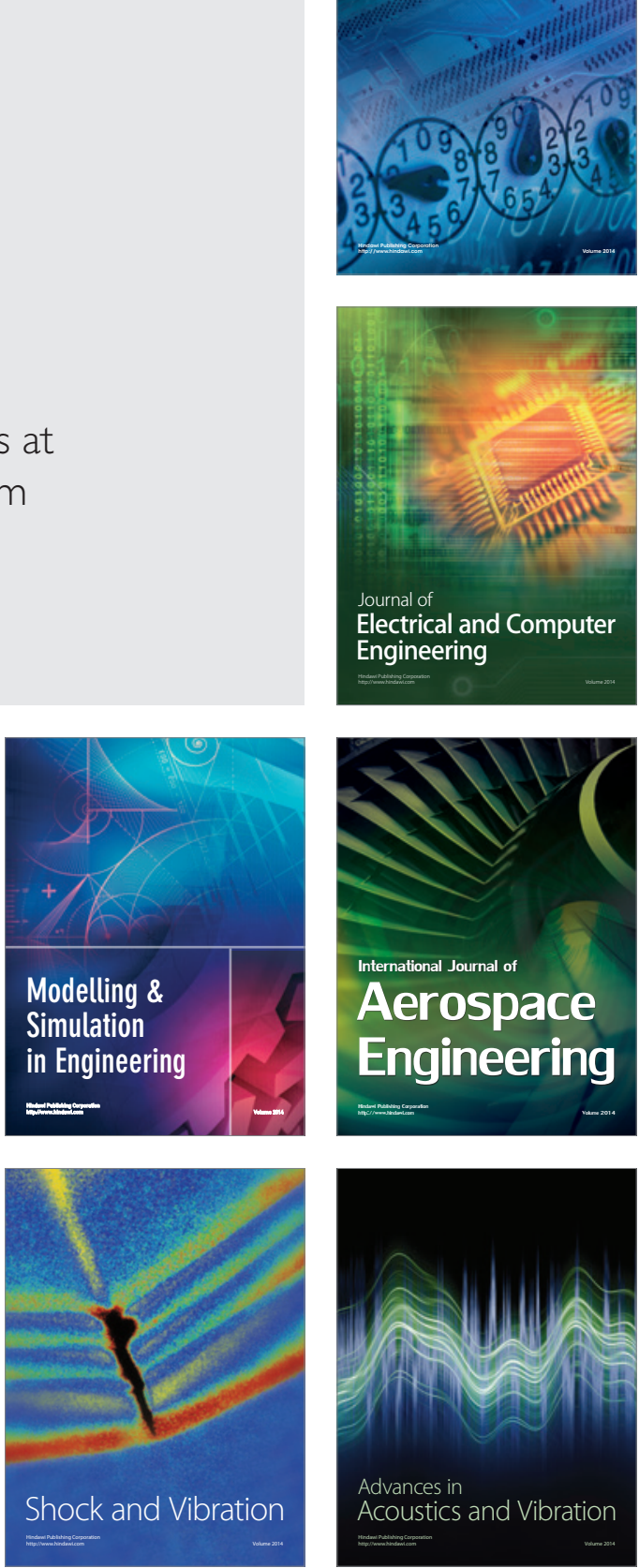